\title{
InterCriteria Analysis of oncological data of the patients for the city of Burgas
}

\author{
Evdokia Sotirova $^{1}$, Yaroslava Petrova ${ }^{2}$ and Hristo Bozov ${ }^{1,2}$ \\ ${ }^{1}$ Faculty of Public Health and Healthcare \\ Prof. Assen Zlatarov University \\ 1 Prof. Yakimov Str, Burgas 8010, Bulgaria \\ e-mail: esotirova@btu.bg \\ ${ }^{2}$ Oncology Complex Center - Burgas \\ 86 Demokratsiya Blvd, Burgas 8000 Bulgaria \\ e-mails: yaroslava_ap@abv.bg, hr_bozov@yahoo.com
}

\begin{abstract}
The InterCriteria Analysis approach is applied to data connected with the field of oncology. The statistical data for newly registered patients with oncological diseases for 2018 in Burgas are studied. The results are commented from different points of view: relations between gender and age of the patients, relations between gender and marital status.
\end{abstract}

Keywords: Intercriteria analysis method, Index matrix, Intuitionistic fuzzy sets, Oncological diseases.

2010 Mathematics Subject Classification: $03 E 72$.

\section{Introduction}

The number of cancer patients in Bulgaria shows a steady upward trend in the last decades [20, 36]. The trend has been marked by more than double the incidence of new cases compared to the 1980s. besides the absolute values, the newly formed cancers occupy an increasing share of the structure of the causes of hospitalization and mortality of the population, as they are second only to the diseases of the blood circulation. Considering the cost of treatment, traceability and prophylaxis, the economic effect on this trend is not to be underestimated either. In this respect, the fact that amongst people with temporary and permanent disability, tumors are the first to be considered, should not be neglected either.

With the advances in invasive cardiology, significant advances in cardiovascular disease prophylaxis and control, and increased public responsibility for this issue, the relative incidence of 
morbidity, mortality, hospitalization and disability in relation to these diseases declines considerably and yields room to neoplasms that take a significant lead.

In summary, these observations on the dynamics of disease causes of public expenditure and severe life-health events, oncological diseases are a major socio-economic problem, which should be a priority. Trend monitoring, dependence, correlation between causal and investigative factors would have a tremendous impact and help in resolving the problems arising from increased oncology morbidity.

For analysis of the data for the patients with oncological diseases, registered in Burgas for 2018, the InterCriteria Analysis (ICA) approach is applied. Iy was introduced by K. Atanassov, D. Mavrov and V. Atanassova in 2014, [6]. The concept of ICA is based on the apparatus of (two-dimensional) Index matrices (IMs, see [4]) and intuitionistic fuzzy sets (IFSs, see [5]).

The approach is especially designed for decision support of multicriteria decision making problems, where some of the criteria have a higher cost than others - for example the harder, more expensive, the ones where more human resource is employed or take more time to measure or evaluate. Although these criteria are seen as more disadvantageous, the method's objective is to discover sufficiently high levels of dependence or correlation between these criteria and others, which are easier, less costly or faster to measure or evaluate, in order to discard the disadvantageous ones from the future decision making process.

After applying the ICA method, we obtain an index matrix that gives the correlations of each pair of criteria presented in the form of intuitionistic fuzzy pairs of values [8].

The dependences between the criteria are called "positive consonance", "negative consonance" or "dissonance". Here we use the scale used in previous studies that is shown in [7].

The ICA approach has been applied for analyzing data and decision making in different areas medical investigations [17, 18, 37, 38, 40, 41], genetic algorithms [1, 2, 3, 21, 23, 26, 29], metaheuristic algorithms [10,11, 12, 13, 14, 15,16, 19, 22, 27, 28, 30, 31], neural networks [32, 33, 34, 35], etc.

In the present paper the ICA method is applied to studying some statistical data for newly registered patients with oncological diseases for 2018 in Burgas. In the next observations we will apply the ICA approach to patients with oncological diseases for 2014-2018 and to data connected to metastatic melanoma [24, 25] and colorectal cancer [39].

\section{Application of the ICA approach}

The InterCriteria Analysis approach is applied to real data for 941 newly registered patients (511 men and 430 women) with oncological diseases for 2018 in Burgas.

The data contains information about age of patients, name of the disease, according to International Statistical Classification of Diseases and Related Health Problems, gender, marital status, data of the registration of the patient, etc. In the observed data there are:

- 16 age groups: men up to 20 years, women up to 20 years, men 21-30, women 21-30, men 31-40, women 31-40, men 41-50, women 41-50, men 51-60, women 51-60, men 61-70, women 61-70, men 71-80, women 71-80, men over 80 , women over 80 . According age groups the patients are distributed the following way: 8 up to 20 years ( 6 men, 2 women); 12 in 21-30 (4 men, 8 women); 25 in 31-40 (9 men, 16 women); 72 in 41-50 (23 men, 49 women); 147 in 51-60 (76 men, 71 women); 303 in 61-70 (189 men, 114 women); 260 in 71-80 (150 men, 110 women); and 114 over 80 (54 men, 60 women);

- 8 marital status groups: men unmarried, women unmarried, men married, women married, men divorced, women divorced, men widower, women widower. According marital status the patients are distributed the following way: 13 unmarried (13 men, 13 women); 718 married (398 men, 320 women); 28 divorced (16 men, 12 women) and 169 married ( 84 men, 85 women). 


\subsection{Applying ICA approach for age and gender data}

We will use an index matrix that contains 16 rows (for age groups and gender) and 4 columns (for marital status). After the applying the ICA method we obtain index matrix (see Table 1) with intuitionistic fuzzy pairs that represents an intuitionistic fuzzy evaluation of the relations between every pair of criteria "age group and gender".

\subsection{Applying ICA approach for marital status and gender data}

We will use an index matrix that contains 8 rows (for marital status and gender) and 8 columns (for age groups). After the applying the ICA method we obtain index matrix (see Table 2) with intuitionistic fuzzy pairs that represents an intuitionistic fuzzy evaluation of the relations between every pair of criteria "marital status and gender". In both cases, the stronger the correlation between a given pair is, the more intense the color is.

\section{Results and discussion}

After applying the ICA approach for age and gender data the following conclusions can be made:

- 9 pairs of criteria (age and gender groups) are in strong positive consonance. In the age groups "up to 20 years", "21-30", "51-60", "61-70" and "over $80 "$ there is a strong positive consonance between gender groups - male and female $\langle 1.000 ; 0.000\rangle$. The age group " $51-60$ " has a strong positive consonance with the age group "61-70", taking gender into account. This shows a constant trend of the patients with oncology diseases for some of the identical or the adjacent age groups, also taking gender into account.

- 16 pairs of criteria (age and gender groups) are in weak positive consonance. In 7 pairs of criteria males in 31-40, 41-50 and 71-80 age groups have similar behavior with females in the same age groups. Males in the 41-50 age group have similar behavior with following age groups: "men 5160", "men 61-70", "women 51-60" and "women 61-70". In next 9 pairs of criteria the intuitionistic fuzzy pairs of age groups show similar behavior with the intuitionistic fuzzy pairs of the next in order adjacent age group.

- 59 pairs of criteria (age and gender groups) are in dissonance. They are mostly made of age groups with a big difference in the years, the lower age groups and the highest age group, regardless of gender.

- The lower and highest age groups have more unstable behavior, while the medium age groups show a higher consistency.

After applying the ICA approach for marital status and gender data the following conclusions can be made:

- The groups of married, widowed and divorced males and females are in positive consonance. This shows a stable tendency regardless of the age group.

- 1 pair of criteria "Men Married - Women Married" is in a strong positive consonance - $\langle 0.964$; $0.000\rangle$. This means that the married men and married women have a very similar tendency for oncological diseases.

- 1 pair of criteria "Men Widowed - Women Widowed" is in a positive consonance - $\langle 0.929 ; 0.071\rangle$. This means that the incidence of cancers in male widows is similar to that of female widows. 
Table 1. Intuitionistic fuzzy pairs of the relations between "age groups and gender group"

\begin{tabular}{|c|c|c|c|c|c|c|c|c|c|c|c|c|c|c|c|c|}
\hline & $\begin{array}{l}\text { men } \\
0-20\end{array}$ & $\begin{array}{c}\text { women } \\
0-20\end{array}$ & $\begin{array}{c}\text { men } \\
21-30\end{array}$ & $\begin{array}{c}\text { women } \\
21-30\end{array}$ & $\begin{array}{c}\text { men } \\
31-40\end{array}$ & $\begin{array}{c}\text { women } \\
31-40\end{array}$ & $\begin{array}{c}\text { men } \\
41-50\end{array}$ & $\begin{array}{c}\text { women } \\
41-50\end{array}$ & $\begin{array}{c}\text { men } \\
51-60\end{array}$ & $\begin{array}{c}\text { women } \\
51-60\end{array}$ & $\begin{array}{c}\text { men } \\
61-70\end{array}$ & $\begin{array}{c}\text { women } \\
61-70\end{array}$ & $\begin{array}{c}\text { men } \\
71-80\end{array}$ & $\begin{array}{c}\text { women } \\
71-80\end{array}$ & \begin{tabular}{|c|} 
men over \\
80
\end{tabular} & $\begin{array}{l}\text { women } \\
\text { over } 80\end{array}$ \\
\hline $\begin{array}{l}\text { men } \\
0-20\end{array}$ & $\begin{array}{l}\langle 1.00 \\
0.00\rangle\end{array}$ & $\begin{array}{l}\langle 1.00 \\
0.00\rangle\end{array}$ & $\begin{array}{l}\langle 0.67 \\
0.00\rangle\end{array}$ & $\begin{array}{l}\langle 0.67 \\
0.00\rangle\end{array}$ & $\begin{array}{c}\langle 0.33 ; \\
0.17\rangle\end{array}$ & $\begin{array}{l}\langle 0.50 \\
0.17\rangle\end{array}$ & $\begin{array}{c}\langle 0.00 ; \\
0.33\rangle\end{array}$ & $\begin{array}{c}\langle 0.17 \\
0.33\rangle\end{array}$ & $\begin{array}{l}\langle 0.00 \\
0.50\rangle\end{array}$ & $\begin{array}{c}\langle 0.00 \\
0.50\rangle\end{array}$ & $\begin{array}{c}\langle 0.00 \\
0.50\rangle\end{array}$ & $\begin{array}{c}\langle 0.00 \\
0.50\rangle\end{array}$ & $\begin{array}{l}\langle 0.00 \\
0.33\rangle\end{array}$ & $\begin{array}{l}\langle 0.17 \\
0.33\rangle\end{array}$ & $\begin{array}{l}\langle 0.00 \\
0.33\rangle\end{array}$ & $\begin{array}{l}\langle 0.00 \\
0.33\rangle\end{array}$ \\
\hline $\begin{array}{c}\text { women } \\
0-20\end{array}$ & $\begin{array}{c}\langle 1.00 \\
0.00\rangle\end{array}$ & $\begin{array}{l}\langle 1.00 ; \\
0.00\rangle\end{array}$ & $\begin{array}{c}\langle 0.67 ; \\
0.00\rangle\end{array}$ & $\begin{array}{l}\langle 0.67 ; \\
0.00\rangle\end{array}$ & $\begin{array}{c}\langle 0.33 ; \\
0.17\rangle\end{array}$ & $\begin{array}{c}\langle 0.50 \\
0.17\rangle\end{array}$ & $\begin{array}{c}\langle 0.00 ; \\
0.33\rangle\end{array}$ & $\begin{array}{l}\langle 0.17 ; \\
0.33\rangle\end{array}$ & $\begin{array}{l}\langle 0.00 \\
0.50\rangle\end{array}$ & $\begin{array}{c}0.00 \\
0.50\rangle\end{array}$ & $\begin{array}{c}\langle 0.00 \\
0.50\rangle\end{array}$ & $\begin{array}{c}\langle 0.00 \\
0.50\rangle\end{array}$ & $\begin{array}{l}\langle 0.00 \\
0.33\rangle\end{array}$ & $\begin{array}{c}\langle 0.17 \\
0.33\rangle\end{array}$ & $\begin{array}{l}0.00 \\
0.33\rangle\end{array}$ & $\begin{array}{l}\langle 0.00 ; \\
0.33\rangle\end{array}$ \\
\hline $\begin{array}{c}\text { men } \\
21-30\end{array}$ & $\begin{array}{c}\langle 0.67 \\
0.00\rangle\end{array}$ & $\begin{array}{l}\langle 0.67 \\
0.00\rangle\end{array}$ & $\begin{array}{c}\langle 1.00 \\
0.00\rangle\end{array}$ & $\begin{array}{c}\langle 1.00 \\
0.00\rangle\end{array}$ & $\begin{array}{l}\langle 0.67 ; \\
0.17\rangle\end{array}$ & $\begin{array}{c}\langle 0.83 \\
0.17\rangle\end{array}$ & $\begin{array}{l}\langle 0.33 ; \\
0.33\rangle\end{array}$ & $\begin{array}{l}\langle 0.50 \\
0.33\rangle\end{array}$ & $\begin{array}{c}\langle 0.33 \\
0.50\rangle\end{array}$ & $\begin{array}{l}\langle 0.33 \\
0.50\rangle\end{array}$ & $\begin{array}{l}\langle 0.33 ; \\
0.50\rangle\end{array}$ & $\begin{array}{c}\langle 0.33 \\
0.50\rangle\end{array}$ & $\begin{array}{l}\langle 0.33 ; \\
0.33\rangle\end{array}$ & $\begin{array}{l}\langle 0.50 \\
0.33\rangle\end{array}$ & $\begin{array}{l}\langle 0.17 ; \\
0.50\rangle\end{array}$ & $\begin{array}{l}\langle 0.17 \\
0.50\rangle\end{array}$ \\
\hline $\begin{array}{c}\text { women } \\
21-30\end{array}$ & $\begin{array}{c}\langle 0.67 \\
0.00\rangle\end{array}$ & $\begin{array}{l}\langle 0.67 \\
0.00\rangle\end{array}$ & $\begin{array}{l}\langle 1.00 \\
0.00\rangle\end{array}$ & $\begin{array}{l}\langle 1.00 \\
0.00\rangle\end{array}$ & $\begin{array}{l}\langle 0.67 ; \\
0.17\rangle\end{array}$ & $\begin{array}{c}\langle 0.83 \\
0.17\rangle\end{array}$ & $\begin{array}{l}\langle 0.33 ; \\
0.33\rangle\end{array}$ & $\begin{array}{l}\langle 0.50 \\
0.33\rangle\end{array}$ & $\begin{array}{c}\langle 0.33 \\
0.50\rangle\end{array}$ & $\begin{array}{l}\langle 0.33 \\
0.50\rangle\end{array}$ & $\begin{array}{l}\langle 0.33 ; \\
0.50\rangle\end{array}$ & $\begin{array}{l}\langle 0.33 \\
0.50\rangle\end{array}$ & $\begin{array}{l}\langle 0.33 ; \\
0.33\rangle\end{array}$ & $\begin{array}{l}\langle 0.50 \\
0.33\rangle\end{array}$ & $\begin{array}{l}\langle 0.17 \\
0.50\rangle\end{array}$ & $\begin{array}{l}\langle 0.17 \\
0.50\rangle\end{array}$ \\
\hline $\begin{array}{c}\text { men } \\
31-40\end{array}$ & $\begin{array}{l}\langle 0.33 \\
0.17\rangle\end{array}$ & $\begin{array}{l}\langle 0.33 ; \\
0.17\rangle\end{array}$ & $\begin{array}{c}\langle 0.67 \\
0.17\rangle\end{array}$ & $\begin{array}{l}\langle 0.67 \\
0.17\rangle\end{array}$ & $\begin{array}{l}\langle 1.00 \\
0.00\rangle\end{array}$ & $\begin{array}{c}\langle 0.83 \\
0.00\rangle\end{array}$ & $\begin{array}{l}\langle 0.67 \\
0.17\rangle\end{array}$ & $\begin{array}{l}0.83 ; \\
0.17\rangle\end{array}$ & $\begin{array}{l}\langle 0.67 ; \\
0.33\rangle\end{array}$ & $\begin{array}{c}\langle 0.67 \\
0.33\rangle\end{array}$ & $\begin{array}{l}0.67 ; \\
0.33\rangle\end{array}$ & $\begin{array}{c}0.67 ; \\
0.33\rangle\end{array}$ & $\begin{array}{l}\langle 0.50 \\
0.33\rangle\end{array}$ & $\begin{array}{l}0.67 ; \\
0.33\rangle\end{array}$ & $\begin{array}{l}\langle 0.33 \\
0.50\rangle\end{array}$ & $\begin{array}{l}0.33 ; \\
0.50\rangle\end{array}$ \\
\hline $\begin{array}{c}\text { women } \\
31-40\end{array}$ & $\begin{array}{c}\langle 0.50 \\
0.17\rangle\end{array}$ & $\begin{array}{l}\langle 0.50 \\
0.17\rangle\end{array}$ & $\begin{array}{l}\langle 0.83 ; \\
0.17\rangle\end{array}$ & $\begin{array}{l}\langle 0.83 \\
0.17\rangle\end{array}$ & $\begin{array}{l}\langle 0.83 ; \\
0.00\rangle\end{array}$ & $\begin{array}{c}\langle 1.00 ; \\
0.00\rangle\end{array}$ & $\begin{array}{l}\langle 0.50 \\
0.17\rangle\end{array}$ & $\begin{array}{l}\langle 0.67 \\
0.17\rangle\end{array}$ & $\begin{array}{c}\langle 0.50 \\
0.33\rangle\end{array}$ & $\begin{array}{c}\langle 0.50 \\
0.33\rangle\end{array}$ & $\begin{array}{c}\langle 0.50 \\
0.33\rangle\end{array}$ & & $\begin{array}{l}\langle 0.50 \\
0.17\rangle\end{array}$ & $\begin{array}{l}\langle 0.67 \\
0.17\rangle\end{array}$ & $\begin{array}{l}\langle 0.33 ; \\
0.33\rangle\end{array}$ & $\begin{array}{l}\langle 0.33 \\
0.33\rangle\end{array}$ \\
\hline $\begin{array}{c}\text { men } \\
41-50\end{array}$ & $\begin{array}{c}\langle 0.00 \\
0.33\rangle\end{array}$ & $\begin{array}{l}\langle 0.00 \\
0.33\rangle\end{array}$ & $\begin{array}{l}\langle 0.33 \\
0.33\rangle\end{array}$ & $\begin{array}{l}\langle 0.33 \\
0.33\rangle\end{array}$ & $\begin{array}{c}\langle 0.67 ; \\
0.17\rangle\end{array}$ & $\begin{array}{c}\langle 0.50 \\
0.17\rangle\end{array}$ & $\begin{array}{l}\langle 1.00 \\
0.00\rangle\end{array}$ & $\begin{array}{l}\langle 0.83 \\
0.00\rangle\end{array}$ & $\begin{array}{c}\langle 0.83 \\
0.00\rangle\end{array}$ & $\begin{array}{c}\langle 0.83 \\
0.00\rangle\end{array}$ & $\begin{array}{l}\langle 0.83 \\
0.00\rangle\end{array}$ & $\begin{array}{c}\langle 0.83 \\
0.00\rangle\end{array}$ & $\begin{array}{l}\langle 0.50 \\
0.17\rangle\end{array}$ & $\begin{array}{l}\langle 0.50 \\
0.33\rangle\end{array}$ & $\begin{array}{c}\langle 0.33 \\
0.33\rangle\end{array}$ & $\begin{array}{l}\langle 0.33 \\
0.33\rangle\end{array}$ \\
\hline $\begin{array}{c}\text { women } \\
41-50\end{array}$ & $\begin{array}{c}\langle 0.17 \\
0.33\rangle\end{array}$ & $\begin{array}{l}0.17 \\
0.33\rangle\end{array}$ & $\begin{array}{c}\langle 0.50 \\
0.33\rangle\end{array}$ & $\begin{array}{l}\langle 0.50 \\
0.33\rangle\end{array}$ & $\begin{array}{c}\langle 0.83 ; \\
0.17\rangle\end{array}$ & $\begin{array}{c}\langle 0.67 \\
0.17\rangle\end{array}$ & $\begin{array}{l}\langle 0.83 ; \\
0.00\rangle\end{array}$ & $\begin{array}{l}\langle 1.00 \\
0.00\rangle\end{array}$ & $\begin{array}{c}\langle 0.83 \\
0.17\rangle\end{array}$ & $\begin{array}{c}\langle 0.83 \\
0.17\rangle\end{array}$ & $\begin{array}{c}\langle 0.83 ; \\
0.17\rangle\end{array}$ & $\begin{array}{c}\langle 0.83 \\
0.17\rangle\end{array}$ & $\begin{array}{l}\langle 0.50 \\
0.33\rangle\end{array}$ & $\begin{array}{l}\langle 0.50 \\
0.50\rangle\end{array}$ & $\begin{array}{c}0.33 \\
0.50\rangle\end{array}$ & $\begin{array}{l}\langle 0.33 ; \\
0.50\rangle\end{array}$ \\
\hline $\begin{array}{c}\text { men } \\
51-60\end{array}$ & $\begin{array}{c}\langle 0.00 \\
0.50\rangle\end{array}$ & $\begin{array}{l}\langle 0.00 \\
0.50\rangle\end{array}$ & $\begin{array}{l}\langle 0.33 \\
0.50\rangle\end{array}$ & $\begin{array}{l}\langle 0.33 \\
0.50\rangle\end{array}$ & $\begin{array}{l}\langle 0.67 ; \\
0.33\rangle\end{array}$ & $\begin{array}{c}\langle 0.50 \\
0.33\rangle\end{array}$ & $\begin{array}{l}\langle 0.83 \\
0.00\rangle\end{array}$ & $\begin{array}{l}\langle 0.83 \\
0.17\rangle\end{array}$ & $\begin{array}{l}\langle 1.00 \\
0.00\rangle\end{array}$ & $\begin{array}{c}\langle 1.00 \\
0.00\rangle\end{array}$ & $\begin{array}{l}\langle 1.00 \\
0.00\rangle\end{array}$ & $\begin{array}{c}\langle 1.00 \\
0.00\rangle\end{array}$ & $\begin{array}{l}\langle 0.67 \\
0.17\rangle\end{array}$ & $\begin{array}{l}\langle 0.67 \\
0.33\rangle\end{array}$ & $\begin{array}{c}\langle 0.50 \\
0.33\rangle\end{array}$ & $\begin{array}{l}\langle 0.50 \\
0.33\rangle\end{array}$ \\
\hline $\begin{array}{c}\text { women } \\
51-60\end{array}$ & $\begin{array}{c}\langle 0.00 \\
0.50\rangle\end{array}$ & $\begin{array}{c}\langle 0.00 \\
0.50\rangle\end{array}$ & $\begin{array}{c}\langle 0.33 \\
0.50\rangle\end{array}$ & $\begin{array}{l}\langle 0.33 \\
0.50\rangle\end{array}$ & $\begin{array}{c}\langle 0.67 ; \\
0.33\rangle\end{array}$ & $\begin{array}{c}\langle 0.50 \\
0.33\rangle\end{array}$ & $\begin{array}{l}\langle 0.83 \\
0.00\rangle\end{array}$ & $\begin{array}{c}\langle 0.83 \\
0.17\rangle\end{array}$ & $\begin{array}{c}\langle 1.00 \\
0.00\rangle \\
\end{array}$ & $\begin{array}{c}\langle 1.00 \\
0.00\rangle\end{array}$ & $\begin{array}{c}\langle 1.00 \\
0.00\rangle\end{array}$ & $\begin{array}{c}\langle 1.00 \\
0.00\rangle\end{array}$ & $\begin{array}{l}\langle 0.67 ; \\
0.17\rangle\end{array}$ & $\begin{array}{l}\langle 0.67 \\
0.33\rangle\end{array}$ & $\begin{array}{c}\langle 0.50 \\
0.33\rangle\end{array}$ & $\begin{array}{l}\langle 0.50 \\
0.33\rangle\end{array}$ \\
\hline $\begin{array}{c}\text { men } \\
61-70\end{array}$ & $\begin{array}{c}\langle 0.00 \\
0.50\rangle\end{array}$ & $\begin{array}{l}0.00 \\
0.50\rangle\end{array}$ & $\begin{array}{c}\langle 0.33 \\
0.50\rangle\end{array}$ & $\begin{array}{l}\langle 0.33 \\
0.50\rangle\end{array}$ & $\begin{array}{c}\langle 0.67 ; \\
0.33\rangle\end{array}$ & $\begin{array}{c}\langle 0.50 \\
0.33\rangle\end{array}$ & $\begin{array}{l}\langle 0.83 ; \\
0.00\rangle\end{array}$ & $\begin{array}{l}\langle 0.83 \\
0.17\rangle\end{array}$ & $\begin{array}{c}\langle 1.00 ; \\
0.00\rangle\end{array}$ & $\begin{array}{c}\langle 1.00 ; \\
0.00\rangle\end{array}$ & $\begin{array}{c}\langle 1.00 ; \\
0.00\rangle\end{array}$ & $\begin{array}{c}\langle 1.00 \\
0.00\rangle\end{array}$ & $\begin{array}{l}\langle 0.67 ; \\
0.17\rangle\end{array}$ & $\begin{array}{l}\langle 0.67 ; \\
0.33\rangle\end{array}$ & $\begin{array}{c}0.50 \\
0.33\rangle\end{array}$ & $\begin{array}{l}\langle 0.50 \\
0.33\rangle\end{array}$ \\
\hline $\begin{array}{c}\text { women } \\
61-70\end{array}$ & $\begin{array}{c}\langle 0.00 \\
0.50\rangle\end{array}$ & $\begin{array}{c}\langle 0.00 \\
0.50\rangle\end{array}$ & $\begin{array}{c}\langle 0.33 \\
0.50\rangle\end{array}$ & $\begin{array}{c}\langle 0.33 \\
0.50\rangle\end{array}$ & $\begin{array}{c}\langle 0.67 ; \\
0.33\rangle\end{array}$ & $\begin{array}{c}\langle 0.50 \\
0.33\rangle\end{array}$ & $\begin{array}{c}\langle 0.83 \\
0.00\rangle\end{array}$ & $\begin{array}{c}\langle 0.83 \\
0.17\rangle\end{array}$ & $\begin{array}{c}\langle 1.00 \\
0.00\rangle\end{array}$ & $\begin{array}{c}\langle 1.00 \\
0.00\rangle\end{array}$ & $\begin{array}{c}\langle 1.00 \\
0.00\rangle\end{array}$ & $\begin{array}{c}\langle 1.00 \\
0.00\rangle\end{array}$ & $\begin{array}{c}\langle 0.67 \\
0.17\rangle\end{array}$ & $\begin{array}{c}\langle 0.67 \\
0.33\rangle\end{array}$ & $\begin{array}{c}\langle 0.50 \\
0.33\rangle\end{array}$ & $\begin{array}{l}\langle 0.50 \\
0.33\rangle\end{array}$ \\
\hline $\begin{array}{c}\text { men } \\
71-80 \\
\end{array}$ & $\begin{array}{c}\langle 0.00 \\
0.33\rangle\end{array}$ & $\begin{array}{l}\langle 0.00 \\
0.33\rangle \\
\end{array}$ & $\begin{array}{c}\langle 0.33 \\
0.33\rangle\end{array}$ & $\begin{array}{c}\langle 0.33 \\
0.33\rangle \\
\end{array}$ & $\begin{array}{c}\langle 0.50 \\
0.33\rangle\end{array}$ & $\begin{array}{c}\langle 0.50 \\
0.17\rangle \\
\end{array}$ & $\begin{array}{l}\langle 0.50 \\
0.17\rangle\end{array}$ & $\begin{array}{l}\langle 0.50 \\
0.33\rangle\end{array}$ & $\begin{array}{c}\langle 0.67 \\
0.17\rangle\end{array}$ & $\begin{array}{c}\langle 0.67 ; \\
0.17\rangle \\
\end{array}$ & $\begin{array}{c}\langle 0.67 ; \\
0.17\rangle\end{array}$ & $\begin{array}{c}\langle 0.67 \\
0.17\rangle \\
\end{array}$ & $\begin{array}{l}\langle 1.00 \\
0.00\rangle\end{array}$ & $\begin{array}{l}0.83 \\
0.00\rangle \\
\end{array}$ & $\begin{array}{c}\langle 0.83 \\
0.17\rangle\end{array}$ & $\begin{array}{c}\langle 0.83 \\
0.17\rangle \\
\end{array}$ \\
\hline $\begin{array}{c}\text { women } \\
71-80\end{array}$ & $\begin{array}{c}\langle 0.17 \\
0.33\rangle\end{array}$ & $\begin{array}{l}0.17 \\
0.33\rangle\end{array}$ & $\begin{array}{c}\langle 0.50 \\
0.33\rangle\end{array}$ & $\begin{array}{l}\langle 0.50 \\
0.33\rangle\end{array}$ & $\begin{array}{c}\langle 0.67 \\
0.33\rangle\end{array}$ & $\begin{array}{c}\langle 0.67 \\
0.17\rangle\end{array}$ & $\begin{array}{c}\langle 0.50 \\
0.33\rangle\end{array}$ & $\begin{array}{l}\langle 0.50 \\
0.50\rangle\end{array}$ & $\begin{array}{c}\langle 0.67 \\
0.33\rangle\end{array}$ & $\begin{array}{c}\langle 0.67 \\
0.33\rangle\end{array}$ & $\begin{array}{l}\langle 0.67 \\
0.33\rangle\end{array}$ & $\begin{array}{c}\langle 0.67 \\
0.33\rangle\end{array}$ & $\begin{array}{l}\langle 0.83 \\
0.00\rangle\end{array}$ & $\begin{array}{c}\langle 1.00 \\
0.00\rangle\end{array}$ & $\begin{array}{c}\langle 0.67 \\
0.17\rangle\end{array}$ & $\begin{array}{l}\langle 0.67 \\
0.17\rangle\end{array}$ \\
\hline $\begin{array}{c}\text { men } \\
\text { over } 80\end{array}$ & $\begin{array}{c}\langle 0.00 \\
0.33\rangle\end{array}$ & $\begin{array}{l}\langle 0.00 \\
0.33\rangle\end{array}$ & $\begin{array}{c}\langle 0.17 \\
0.50\rangle\end{array}$ & $\begin{array}{l}\langle 0.17 \\
0.50\rangle\end{array}$ & $\begin{array}{c}\langle 0.33 \\
0.50\rangle\end{array}$ & $\begin{array}{l}\langle 0.33 \\
0.33\rangle\end{array}$ & $\begin{array}{l}\langle 0.33 \\
0.33\rangle\end{array}$ & $\begin{array}{l}\langle 0.33 \\
0.50\rangle\end{array}$ & $\begin{array}{c}\langle 0.50 \\
0.33\rangle\end{array}$ & $\begin{array}{c}\langle 0.50 \\
0.33\rangle\end{array}$ & $\begin{array}{c}\langle 0.50 \\
0.33\rangle\end{array}$ & $\begin{array}{c}\langle 0.50 \\
0.33\rangle\end{array}$ & $\begin{array}{l}\langle 0.83 \\
0.17\rangle\end{array}$ & $\begin{array}{c}\langle 0.67 \\
0.17\rangle\end{array}$ & $\begin{array}{l}\langle 1.00 \\
0.00\rangle\end{array}$ & $\begin{array}{l}\langle 1.00 \\
0.00\rangle\end{array}$ \\
\hline $\begin{array}{l}\text { women } \\
\text { over } 80\end{array}$ & $\begin{array}{c}\langle 0.00 \\
0.33\rangle\end{array}$ & $\begin{array}{l}\langle 0.00 \\
0.33\rangle \\
\end{array}$ & $\begin{array}{c}\langle 0.17 \\
0.50\rangle \\
\end{array}$ & $\begin{array}{l}\langle 0.17 \\
0.50\rangle \\
\end{array}$ & $\begin{array}{c}\langle 0.33 \\
0.50\rangle\end{array}$ & $\begin{array}{l}\langle 0.33 ; \\
0.33\rangle\end{array}$ & $\begin{array}{l}\langle 0.33 ; \\
0.33\rangle\end{array}$ & $\begin{array}{l}\langle 0.33 ; \\
0.50\rangle\end{array}$ & $\begin{array}{l}\langle 0.50 \\
0.33\rangle\end{array}$ & $\begin{array}{c}\langle 0.50 \\
0.33\rangle \\
\end{array}$ & $\begin{array}{c}\langle 0.50 \\
0.33\rangle\end{array}$ & $\begin{array}{c}0.50 ; \\
0.33\rangle \\
\end{array}$ & $\begin{array}{l}\langle 0.83 ; \\
0.17\rangle\end{array}$ & $\begin{array}{l}0.67 \\
0.17\rangle \\
\end{array}$ & $\begin{array}{c}\langle 1.00 ; \\
0.00\rangle\end{array}$ & $\begin{array}{l}\langle 1.00 ; \\
0.00\rangle\end{array}$ \\
\hline
\end{tabular}


Table 2. Intuitionistic fuzzy pairs of the relations between "marital status and gender group"

\begin{tabular}{|l|c|c|c|c|c|c|c|c|}
\hline & $\begin{array}{c}\text { Men } \\
\text { Unmarried }\end{array}$ & $\begin{array}{c}\text { Men } \\
\text { Married }\end{array}$ & $\begin{array}{c}\text { Men } \\
\text { Divorced }\end{array}$ & $\begin{array}{c}\text { Men } \\
\text { Widowed }\end{array}$ & $\begin{array}{c}\text { Women } \\
\text { Unmarried }\end{array}$ & $\begin{array}{c}\text { Women } \\
\text { Married }\end{array}$ & $\begin{array}{c}\text { Women } \\
\text { Divorced }\end{array}$ & $\begin{array}{c}\text { Women } \\
\text { Widowed }\end{array}$ \\
\hline Men & $\langle 1.000 ;$ & $\langle 0.143 ;$ & $\langle 0.214 ;$ & $\langle 0.071 ;$ & $\langle 0.643 ;$ & $\langle 0.143 ;$ & $\langle 0.179 ;$ & $\langle 0.036 ;$ \\
Unmarried & $0.000\rangle$ & $0.607\rangle$ & $0.321\rangle$ & $0.464\rangle$ & $0.036\rangle$ & $0.571\rangle$ & $0.286\rangle$ & $0.500\rangle$ \\
\hline Men & $\langle 0.143 ;$ & $\langle 1.000 ;$ & $\langle 0.571 ;$ & $\langle 0.643 ;$ & $\langle 0.179 ;$ & $\langle 0.964 ;$ & $\langle 0.571 ;$ & $\langle 0.571 ;$ \\
Married & $0.607\rangle$ & $0.000\rangle$ & $0.143\rangle$ & $0.143\rangle$ & $0.464\rangle$ & $0.000\rangle$ & $0.071\rangle$ & $0.214\rangle$ \\
\hline Men & $\langle 0.214 ;$ & $\langle 0.571 ;$ & $\langle 1.000 ;$ & $\langle 0.357 ;$ & $\langle 0.214 ;$ & $\langle 0.571 ;$ & $\langle 0.786 ;$ & $\langle 0.357 ;$ \\
Divorced & $0.321\rangle$ & $0.143\rangle$ & $0.000\rangle$ & $0.286\rangle$ & $0.286\rangle$ & $0.107\rangle$ & $0.000\rangle$ & $0.286\rangle$ \\
\hline Men & $\langle 0.071 ;$ & $\langle 0.643 ;$ & $\langle 0.357 ;$ & $\langle 1.000 ;$ & $\langle 0.071 ;$ & $\langle 0.607 ;$ & $\langle 0.429 ;$ & $\langle 0.929 ;$ \\
Widowed & $0.464\rangle$ & $0.143\rangle$ & $0.286\rangle$ & $0.000\rangle$ & $0.429\rangle$ & $0.143\rangle$ & $0.214\rangle$ & $0.071\rangle$ \\
\hline Women & $\langle 0.643 ;$ & $\langle 0.179 ;$ & $\langle 0.214 ;$ & $\langle 0.071 ;$ & $\langle 1.000 ;$ & $\langle 0.179 ;$ & $\langle 0.143 ;$ & $\langle 0.036 ;$ \\
Unmarried & $0.036\rangle$ & $0.464\rangle$ & $0.286\rangle$ & $0.429\rangle$ & $0.000\rangle$ & $0.429\rangle$ & $0.214\rangle$ & $0.464\rangle$ \\
\hline Women & $\langle 0.143 ;$ & $\langle 0.964 ;$ & $\langle 0.571 ;$ & $\langle 0.607 ;$ & $\langle 0.179 ;$ & $\langle 1.000 ;$ & $\langle 0.607 ;$ & $\langle 0.536 ;$ \\
Married & $0.571\rangle$ & $0.000\rangle$ & $0.107\rangle$ & $0.143\rangle$ & $0.429\rangle$ & $0.000\rangle$ & $0.071\rangle$ & $0.214\rangle$ \\
\hline Women & $\langle 0.179 ;$ & $\langle 0.571 ;$ & $\langle 0.786 ;$ & $\langle 0.429 ;$ & $\langle 0.143 ;$ & $\langle 0.607 ;$ & $\langle 1.000 ;$ & $\langle 0.393 ;$ \\
Divorced & $0.286\rangle$ & $0.071\rangle$ & $0.000\rangle$ & $0.214\rangle$ & $0.214\rangle$ & $0.071\rangle$ & $0.000\rangle$ & $0.250\rangle$ \\
\hline Women & $\langle 0.036 ;$ & $\langle 0.571 ;$ & $\langle 0.357 ;$ & $\langle 0.929 ;$ & $\langle 0.036 ;$ & $\langle 0.536 ;$ & $\langle 0.393 ;$ & $\langle 1.000 ;$ \\
Widowed & $0.500\rangle$ & $0.214\rangle$ & $0.286\rangle$ & $0.071\rangle$ & $0.464\rangle$ & $0.214\rangle$ & $0.250\rangle$ & $0.000\rangle$ \\
\hline
\end{tabular}

- 1 pair of criteria "Men Divorced - Women Divorced" is in a weak positive consonance - $\langle 0.786$; $0.000\rangle$. This means that the trends for cancers in male widows is similar to that of female widows.

- The married, widowed and divorced male and female groups show a clearly expressed tendency for consistency, while the groups of unmarried male and unmarried female have unstable behavior. The groups of unmarried male and unmarried female are in dissonance with each other.

- There are no correlations between different marital status groups, regardless of gender.

\section{Conclusion}

The InterCriteria Analysis method is applied to statistical data for newly registered and dispensary patients with oncological diseases for 2018 in Burgas. The analyzed data are from regional databases.

The results are commented from different points of view: relations between gender and age of the patients, and relations between gender and marital status.

Thus predictors of disease can be generated and search for dependencies between determinants of multi-criteria decision-making in oncological diseases associated with limitations such as time and resources.

In the next paper, we will use the method proposed in [9] for constructing of three two-dimensional Index Matrices from the three-dimensional one aiming at detecting patterns and relationships across the data per oncology patients' profiles, per marital status, and per year. ICA approach will be applied into the next directions: for age and gender group data, for marital status data and by years with collected statistical data for registered patients with oncological diseases in Burgas for 2014-2018. 


\section{Acknowledgements}

This research has been supported by the Bulgarian National Science Fund under Grant Ref. No. KP-06N22/1/2018 "Theoretical research and applications of InterCriteria Analysis".

\section{References}

[1] Angelova, M., Roeva, O., \& Pencheva, T. (2015). InterCriteria Analysis of a Cultivation Process Model Based on the Genetic Algorithm Population Size Influence, Notes on Intuitionistic Fuzzy Sets, 21 (4), 90-103.

[2] Angelova, M., O. Roeva, T. Pencheva, InterCriteria Analysis of Crossover and Mutation Rates Relations in Simple Genetic Algorithm, Annals of Computer Science and Information Systems, Vol. 5, 2015, 419-424.

[3] Angelova, M., T. Pencheva, InterCriteria Analysis of Multi-population Genetic Algorithms Performance, Annals of Computer Science and Information Systems, Vol. 13, 2017, 77-82.

[4] Atanassov, K. Index Matrices: Towards an Augmented Matrix Calculus. Studies in Computational Intelligence Series, Vol. 573, Springer, Cham, 2014.

[5] Atanassov, K. On Intuitionistic Fuzzy Sets Theory, Springer, Berlin, 2012.

[6] Atanassov, K., D. Mavrov, V. Atanassova, Intercriteria Decision Making: A New Approach for Multicriteria Decision Making, Based on Index Matrices and Intuitionistic Fuzzy Sets. Issues in Intuitionistic Fuzzy Sets and Generalized Nets, 11, 2014, 1-8.

[7] Atanassov, K, V. Atanassova, G. Gluhchev, InterCriteria Analysis: Ideas and problems, Notes on Intuitionistic Fuzzy Sets, Vol. 21, No. 1, 2015, 81-88.

[8] Atanassov, K., E. Szmidt, J. Kacprzyk, On intuitionistic fuzzy pairs, Notes on Intuitionistic Fuzzy Sets, 19 (3), 2013, 1-13.

[9] Atanassov, K. (2014). On index matrices. Part 5: 3-dimensional index matrices. Advanced Studies in Contemporary Mathematics, 24 (4), 423-432.

[10] Fidanova, S., \& Roeva, O. (2016). InterCriteria Analysis of Different Metaheuristics Applied to E. coli Cultivation Process. Proc. of Numerical Methods for Scientific Computations and Advanced Applications, May 29- June 02, 2016, Hissarya, 21-24.

[11] Fidanova, S., Roeva, O., Paprzycki, M., \& Gepner, P. InterCriteria Analysis of ACO Start Strategies. Proceedings of the FedCSIS'2016, 547-550.

[12] Fidanova, S., Roeva, O., Mucherino, A., \& Kapanova, K. (2016). InterCriteria Analysis of Ant Algorithm with Environment Change for GPS Surveying Problem. Lecture Notes in Computer Science, 9883, Springer, 271-278.

[13] Fidanova, S., \& Roeva, O. (2018). Comparison of Different Metaheuristic Algorithms based on InterCriteria Analysis, Journal of Computational and Applied Mathematics, 340, 615-628.

[14] Fidanova, S., O. Roeva, M. Paprzycki, InterCriteria Analysis of Ant Colony Optimization Application to GPS Surveying Problems, Issues in Intuitionistic Fuzzy Sets and Generalized Nets, Vol. 12, 2015/2016, 20-38. 
[15] Fidanova S., \& Roeva, O. (2019). InterCriteria Analysis of Different Variants of ACO algorithm for Wireless Sensor Network Positioning, Lecture Notes in Computer Science, Vol. 11189, 88-96.

[16] Fidanova, S., Atanassova, V., \& Roeva, O. (2017). Ant Colony Optimization Application to GPS Surveying Problems: InterCriteria Analysis, Uncertainty and Imprecision in Decision Making and Decision Support: Cross Fertilization, New Models and Applications, Advances in Intelligent Systems and Computing, Springer, Vol. 559, 251-264.

[17] Ilkova, T., Roeva, O., Vassilev, P., \& Petrov, M. (2015/2016) InterCriteria Analysis in Structural and Parameter Identification of L-lysine Production Model. Issues in Intuitionistic Fuzzy Sets and Generalized Nets, 12, 39-52.

[18] Krumova, S., Todinova, S., Mavrov, D., Marinov, P., Atanassova, V., Atanassov, K., \& Taneva, S. (2017). Intercriteria analysis of calorimetric data of blood serum proteome. Biochimica et Biophysica Acta - General Subjects, 1861 (2), 409-417.

[19] Mucherino, A., Fidanova, S., \& Ganzha, M. (2016). Introducing the Environment in Ant Colony Optimization. Springer Studies of Computational Intelligence, Vol. 655, Springer, 147-158.

[20] National Center of Public Health and Analyses, Annual information, Retrieved from: http://ncpha.government.bg/index.php?lang=en.

[21] Pencheva, T. \& Angelova, M. (2017). InterCriteria Analysis of Simple Genetic Algorithms Performance, Chapter in: Advanced Computing in Industrial Mathematics, Studies in Computational Intelligence, Vol. 681, 147-159.

[22] Pencheva, T., Angelova, M., Vassilev, P., \& Roeva, O. (2016). InterCriteria Analysis Approach to Parameter Identification of a Fermentation Process Model, In: Novel Developments in Uncertainty Representation and Processing, Part V, Vol. 401, Advances in Intelligent Systems and Computing, 385-397.

[23] Pencheva, T., Roeva, O., \& Angelova, M. (2018). Investigation of Genetic Algorithm Performance Based on Different Algorithms for InterCriteria Relations Calculation, Lecture Notes in Computer Science, Vol. 10665, Springer, Cham, 2018, 390-398.

[24] Popov, I., Zheliazkov, R., Ivanova, D., \& Shomov, G. (2015). Metastatic Melanoma and Kidneys, Nephrology, Dialysis and Transplantation, 21 (3), 21-24.

[25] Popov, I., Zheliazkov, R., Ivanova, D., \& Shomov, G. (2015). Metastatic Melanoma - Kidneys, Myocard, Lungs and Liver, Spleen. Clinical case. Nephrology, Dialysis and Transplantation, $21(2), 9$.

[26] Roeva, O., Vassilev, P., Fidanova, S., \& Paprzycki, M. (2016). InterCriteria Analysis of Genetic Algorithms Performance. Studies of Computational Intelligence, Vol. 655, Springer, 235-260.

[27] Roeva, O., Perez, J., Valdez, F., \& Castillo, O. (2016). InterCriteria Analysis of Bat Algorithm with Parameter Adaptation Using Type-1 and Interval Type-2 Fuzzy Systems, Notes on Intuitionistic Fuzzy Sets, 22 (3), 91-105.

[28] Roeva, O., \& Zoteva, D. (2018). Knowledge discovery from data: InterCriteria Analysis of mutation rate influence, Notes on Intuitionistic Fuzzy Sets, 24 (1), 120-130.

[29] Roeva, O., \& Vassilev, P. (2016). InterCriteria Analysis of Generation Gap Influence on Genetic Algorithms Performance, In: Novel Developments in Uncertainty Representation and Processing, Part V, (K. T. Atanassov, O. Castillo, J. Kacprzyk, M. Krawczak, P. Melin, S. Sotirov, E. Sotirova, 
E. Szmidt, G. De Tré, S. Zadrożny, Eds.), Vol. 401, Advances in Intelligent Systems and Computing, Springer, 301-313.

[30] Roeva, O., Fidanova, S., \& Paprzycki, M. (2018). Comparison of Different ACO Start Strategies Based on InterCriteria Analysis, Recent Advances in Computational Optimization, Studies in Computational Intelligence, Vol. 717, Springer, 53-72.

[31] Roeva, O., Fidanova, S., \& Paprzycki, M. (2016). InterCriteria Analysis of ACO and GA Hybrid Algorithms, In: Fidanova S. (eds) Recent Advances in Computational Optimization. Studies in Computational Intelligence, Vol. 610, Springer, 107-126.

[32] Sotirov, S., Sotirova, E., Melin, P., Castillo, O., \& Atanassov, K. (2015). Modular Neural Network Preprocessing Procedure with Intuitionistic Fuzzy InterCriteria Analysis Method, In:- Proc. of Flexible Query Answering Systems (FQAS'2015), Advances in Intelligent Systems and Computing, Vol. 400, Springer International Publishing, 175-186.

[33] Sotirov, S. (2015). Opportunities for application of the intercriteria analysis method to neural network preprocessing procedures, Notes on Intuitionistic Fuzzy Sets, 21 (4), 143-152.

[34] Sotirov, S., Atanassova, V., Sotirova, E., Doukovska, L., Bureva, V., Mavrov, D., \& Tomov, J. (2017). Application of the Intuitionistic Fuzzy InterCriteria Analysis Method with triples to a Neural Network Preprocessing Procedure, Computational Intelligence and Neuroscience, Hindawi, 2017, Article ID 2157852, 2017, 9 pages.

[35] Sotirov, S., Atanassova, V., Sotirova, E., Bureva, V., \& Mavrov, D. (2015). Application of the Intuitionistic Fuzzy InterCriteria Analysis Method to a Neural Network Preprocessing Procedure, Proc. of 9th Conference of the European Society for Fuzzy Logic and Technology (EUSFLAT), 30.06-03.07.2015, Gijon, Spain, 1559-1564.

[36] OECD/European Observatory on Health Systems and Policies (2017). България: Здравен профил за страната 2017, State of Health in the EU, OECD Publishing, Paris/European Observatory on Health Systems and Policies, Brussels. http://dx.doi.org/10.1787/9789264285071-bg (in Bulgarian)

[37] Todinova, S., Mavrov, D., Krumova, S., Marinov, P., Atanassova, V., Atanassov, K., \& Taneva. S. G. (2016). Blood Plasma Thermograms Dataset Analysis by Means of InterCriteria and Correlation Analyses for the Case of Colorectal Cancer. Int. J. Bioautomation, 20 (1), 115-124.

[38] Vankova, D., Sotirova, E., \& Bureva, V. (2015). An application of the InterCriteria Analysis approach to health-related quality of life, Notes on Intuitionistic Fuzzy Sets, 21 (5), 40-48.

[39] Vassilev, V., Tanev, D., Kavrakov, T., \& Abrashev, H. (2015). Our experience in surgical treatment of the complicated forms of colorectal cancer, Trakia Journal of Sciences, 4, 68-70.

[40] Zaharieva, B., Doukovska, L., Ribagin, S., Michalikova, A., \& Radeva, I. (2017). Intercriteria Analysis of Behterev's Kinesitherapy Program, Notes on Intuitionistic Fuzzy Sets, 23 (3), 69-80.

[41] Zaharieva, B., Doukovska, L. Ribagin, S., \& Radeva, I. (2017). InterCriteria approach to Behterev's disease analysis, Notes on Intuitionistic Fuzzy Sets, 23 (2), 119-127. 